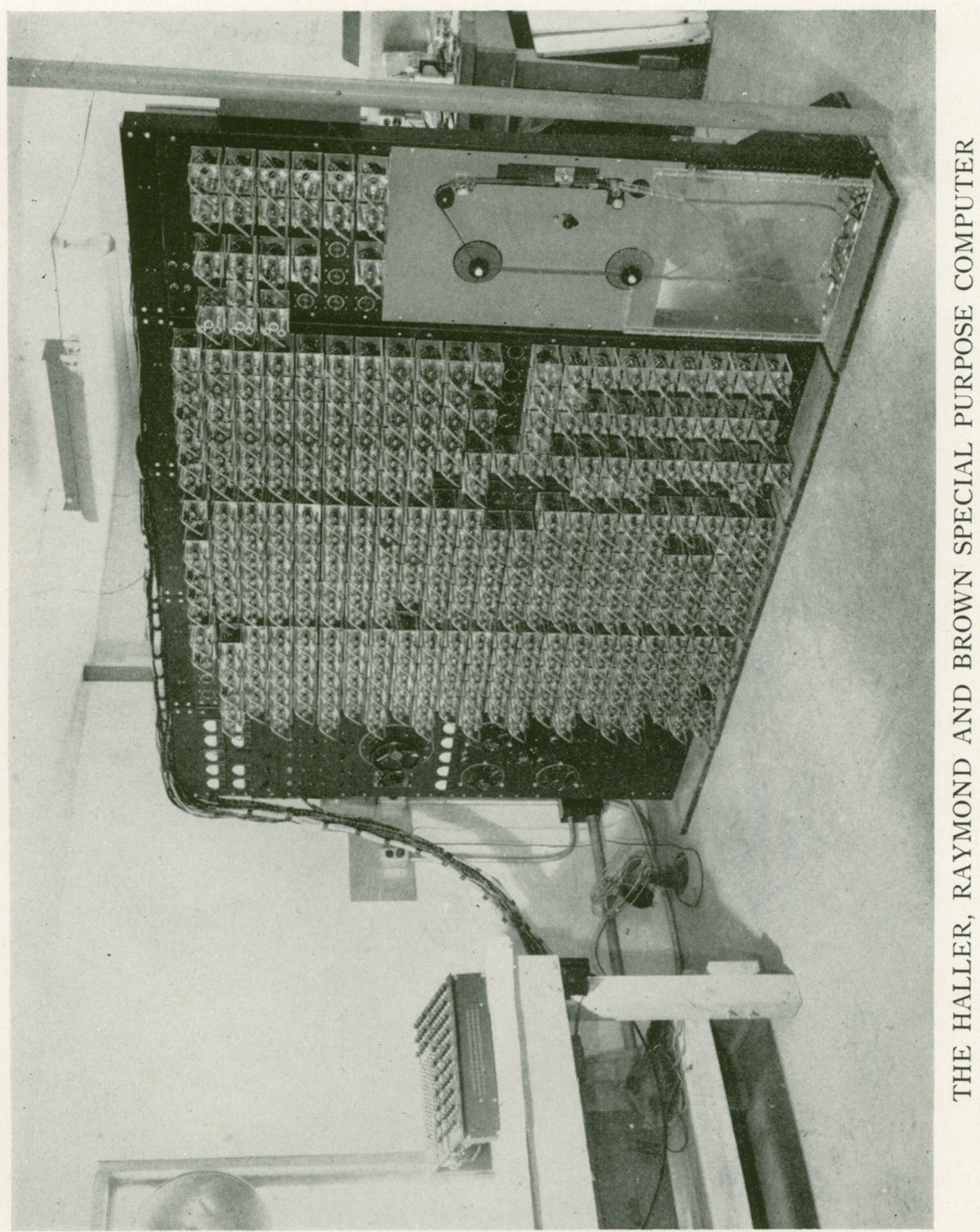




\section{Stability Conditions in the Numerical Treatment of Parabolic Differential Equations}

1. Introduction. The numerical solution of a hyperbolic partial difference equation is in some circumstances subject to an "instability" which has been shown by Lewy and others ${ }^{1}$ to have a simple significance with reference to the differential equation which the difference equation approximates. For example, the difference equation

$$
\left(\phi_{n, j+1}-2 \phi_{n, j}+\phi_{n, j-1}\right) /(\Delta t)^{2}=c^{2}\left(\phi_{n+1, j}-2 \phi_{n, j}+\phi_{n-1, j}\right) /(\Delta x)^{2}
$$

may be used to approximate the wave equation

$$
\phi_{t t}=c^{2} \phi_{x x} \text {. }
$$

If initial values, $\phi_{n, 0}$ and $\phi_{n, 1}$, are specified (corresponding to the specification of $\phi(x, 0)$ and $\left.\phi_{t}(x, 0)\right)$, equation (1) may be used to evaluate first $\phi_{n, 2}$, then $\phi_{n, 3}$, etc. If a "component" of $\phi_{n, j}$, i.e. a perturbation to $\phi_{n, j}$ which preserves the satisfaction of the difference equation and its boundary specifications other than initial values, varies with $n$ as $(-1)^{n}$ (i.e. with the shortest wavelength which can be represented with the lattice spacing $\Delta x$ ) and depends upon $j$ as $K^{j}$, then from (1)

$$
K^{2}-2 K+1=-4 \sigma K ; \quad \sigma=(c \Delta t / \Delta x)^{2} .
$$

If $\sigma>1$, equation (3) has one real root of magnitude greater than unity; hence this (shortest wavelength) component of $\phi$ grows exponentially with $j$. Thus if this component is minutely excited, say through rounding errors, its exponential growth may, for sufficiently large $j$, destroy the resemblance of $\phi_{n, j}$ to the solution of (2). The possibility of such an uncontrolled growth of error is termed "instability," its absence "stability." For $\sigma \leqslant 1$ no such instability occurs.

The significance of the abrupt change in behavior of (1) as $c \Delta t$ exceeds $\Delta x$ may be seen as follows: Each $\phi_{n, j}$ is computed by (1) with the use of preceding values, $\phi_{n, j-1}$ and $\phi_{n, j-2}$, and the neighboring values, $\phi_{n-1, j-1}$ and $\phi_{n+1, j-1}$. Thus $\phi_{n, j}$ is subject to influence only from within a "generating cone" described by $j^{\prime}<j,\left|n-n^{\prime}\right| \leqslant\left|j-j^{\prime}\right|$. It is ultimately determined by those initial values $\phi_{n}{ }^{\prime}, 0$ for which $\left|n^{\prime}-n\right| \leqslant j$. In the difference equation the propagation of influences is thus limited to rates $\leqslant \Delta x / \Delta t$. The differential equation (2) represents the propagation of influence along the characteristic lines, $x \pm c t=$ constant; hence cannot be well represented by (1) unless $\Delta x / \Delta t$ is at least as great as $c$. A more general study of hyperbolic difference equations shows the presence of instability whenever the mesh ratio $\Delta x / \Delta t$ is too small to permit the propagation of influence along the characteristics of the corresponding differential equation.

In the solution of parabolic difference equations stability conditions depending on the mesh intervals may also arise. They are considerably more burdensome than the above condition, usually requiring that $\Delta t$ be small of 
the order of $(\Delta x) .{ }^{2}$ By suitably modifying the difference equation, the limitation imposed by the stability condition can be removed. The character of the diffusion process described by a parabolic equation suggests that a stability condition which limits $\Delta t /(\Delta x)^{2}$ or even $\Delta t / \Delta x$ is not fundamental. The effects of a localized disturbance are appreciable within a range which increases with elapsed time, $t$, about as $t^{\frac{1}{1}}$. Thus the region of appreciable influence can asymptotically be included within an arbitrarily narrow generating cone.

In sections 2 and 3 the stability condition limiting the time interval in a conventional treatment of a simple parabolic differential equation is displayed and its burdensome character illustrated.

In section 4 an alternative simple parabolic difference equation approximating the same differential equation is shown to be stable for all values of $\Delta t /(\Delta x)^{2}$. It is shown in section 5 that the condition for convergence to the solution of the corresponding parabolic differential equation is $\Delta x \rightarrow 0$, $\Delta t / \Delta x \rightarrow 0$. In sections 7 and 8 various generalizations of this method of calculation are examined, particularly with reference to their stability. In these generalizations the definition of "instability" given above becomes inapplicable. The term will there be used in a looser sense to indicate a tendency toward the exaggeration of initially small "errors." Accordingly the indications of stability given there are plausibility-proofs rather than rigorous arguments.

2. Interval limitation for a one-dimensional diffusion equation. The diffusion equation

$$
\phi_{t}=p(x) \phi_{x x} ; \quad p(x)>0
$$

may be approximated by the difference equation

$$
\left(\phi_{n, j+1}-\phi_{n, j}\right) / \Delta t=p_{n}\left(\phi_{n-1, j}-2 \phi_{n, j}+\phi_{n+1, j}\right) / \Delta x^{2} ; \quad p_{n}=p(n \Delta x) .
$$

If initial vaues $\phi_{n, 0}$ are specified, equation (5) permits the successive evaluation of $\phi_{n, 1}, \phi_{n, 2}$, etc. An approximate stability condition for this process is readily derived by the assumption that $p_{n}$ changes slowly with $n$. An alternating component of $\phi_{n, j}, \phi_{n, j} \sim(-1)^{n}$, then appears in $\phi_{n, j+1}$ modified by the factor $1-4 \Delta t p_{n} / \Delta x^{2}$,

$$
\phi_{n, j+1} \sim\left(1-4 \sigma p_{n}\right) \phi_{n, j} ; \quad \sigma=\Delta t / \Delta x^{2} .
$$

An exponential increase of this alternating component may be expected if $p_{n}>1 / 2 \sigma$ over any appreciable region. The condition for stability is then

$$
\sigma \leqslant 1 / 2 p_{M}
$$

where $p_{M}$ approximates the maximum of the $p_{n}$. More precisely, the procedure described by (5) is unstable if an error in $\phi_{n, j}$ recurs in the same form in $\phi_{n, j+1}$ with increased magnitude, i.e. if an error component

$$
\delta \phi_{n j}=K^{i} \lambda_{n} ;|K|>1
$$

satisfies (5) and $\lambda_{n}$ satisfies homogeneous boundary conditions corresponding to those imposed on $\phi_{n, j}$. For definiteness it may be assumed that $\phi_{0, j}$ and $\phi_{N, j}$ are specified. Then an "error-eigenfunction," $\lambda_{n}$, must satisfy

$$
-4 p \lambda_{n}=(K-1) \lambda_{n} / \sigma=p_{n}\left(\lambda_{n-1}-2 \lambda_{n}+\lambda_{n+1}\right) ; \quad \lambda_{0}=\lambda_{N}=0 .
$$


Multiplying by $\lambda_{n} / p_{n}$ and summing yields

$$
\begin{aligned}
-4 p \sum_{n=0}^{N} \lambda_{n}{ }^{2} / p_{n} & =\sum_{n=0}^{N}\left[\lambda_{n}\left(\lambda_{n+1}-\lambda_{n}\right)-\lambda_{n}\left(\lambda_{n}-\lambda_{n-1}\right)\right] \\
& =\sum_{n=0}^{N-1} \lambda_{n}\left(\lambda_{n+1}-\lambda_{n}\right)-\sum_{n=0}^{N-1} \lambda_{n+1}\left(\lambda_{n+1}-\lambda_{n}\right) \\
& =-\sum_{n=0}^{N-1}\left(\lambda_{n+1}-\lambda_{n}\right)^{2}<0 ;
\end{aligned}
$$

thus the positiveness of an eigenvalue, $p$, follows from the assumed positiveness of $p_{n}$. This requires that $K<1$; hence instability can arise only if for some error-eigenfunction $K<-1$. The stability condition (6) holds strictly if $p_{M}$ denotes the greatest eigenvalue, $p$, of (7).

The stability condition (6) imposes a burdensome limitation on the numerical treatment of parabolic differential equations by this method since a moderately fine spatial division may require an immoderately fine temporal division. This will particularly be the case if a very fine structural representation is required (large $N$ ) or if a great disparity exists between the largest and smallest values of $p_{n}$. If the range in $t$ for which a solution is required is of the order of the time required for effective diffusion to the distance $N \Delta x$, the number of time intervals required will be of the order of

$$
t_{\max } / \Delta t \sim N^{2} p_{M} / p_{0}
$$

where $p_{0}$ approximates the smallest of the $p_{n}$.

3. Application to the radial diffusion equation. To illustrate the above general conditions we consider the simple diffusion equation

$$
\begin{array}{cl}
\phi_{t}=\phi_{r r}+\phi_{r} / r ; & \phi(r, 0)=\Phi(r) \\
& \phi(1, t)=a \\
& \phi(R, t)=b .
\end{array}
$$

Writing $r=e^{x}$ puts (8) in the form

$$
\begin{array}{ll}
\phi_{t}=e^{-2 x} \phi_{x x} ; & \phi(x, 0)=\Phi(x) \\
& \phi(0, t)=a \\
& \phi(\ln R, t)=b .
\end{array}
$$

This is of the form of equation (4) with $p(x)=e^{-2 x}$. The error-eigenfunction equation (7) is now

$$
4 p \eta_{n}=(1-K) \eta_{n} / \sigma=e^{-2 n \Delta x}\left(\eta_{n+1}+2 \eta_{n}+\eta_{n-1}\right) ; \quad \eta_{0}=\eta_{N}=0,
$$
where $\eta_{n}=(-1)^{n} \lambda_{n}$.

The greatest eigenvalue, $p_{M}$, is readily approximated by a variation principle:

For each solution of (10) the expression

$$
\chi=2 \sum_{n=0}^{N-1} \eta_{n}\left(\eta_{n}+\eta_{n+1}\right) / \sum_{0}^{N} \eta_{n}^{2} e^{2 n \Delta x}
$$

takes on a stationary value, namely $4 p$. 
The maximum value permitted to $\chi$ is then

$$
\chi_{M}=4 p_{M}=\left(1-K_{\min }\right) / \sigma .
$$

The condition for stability, $K_{\min } \geqslant-1$, then requires

$$
\sigma \leqslant 2 / \chi_{M} \equiv \sigma_{\text {crit }} \text {. }
$$

A lower bound to $\chi_{M}$, hence an upper bound to $\sigma_{\text {crit }}$ (for $N=\infty$ ) has been obtained by setting $\eta_{n}=n e^{-\beta n}$ and maximizing $\chi$ with respect to $\beta$. The resulting approximation to $\sigma_{\text {crit }}$ is displayed (vs. $\Delta x$ ) in Fig. 1 . To estimate the error in this approximation numerical solutions of the difference equations for two values of $\Delta x$ were carried out with excessive $\sigma$ 's. The rates of growth of the dominant instabilities determine $\chi_{M}$ (by eq. 11), hence $\sigma_{\text {crit }}$. These two "experimental" values are also shown in Fig. 1.

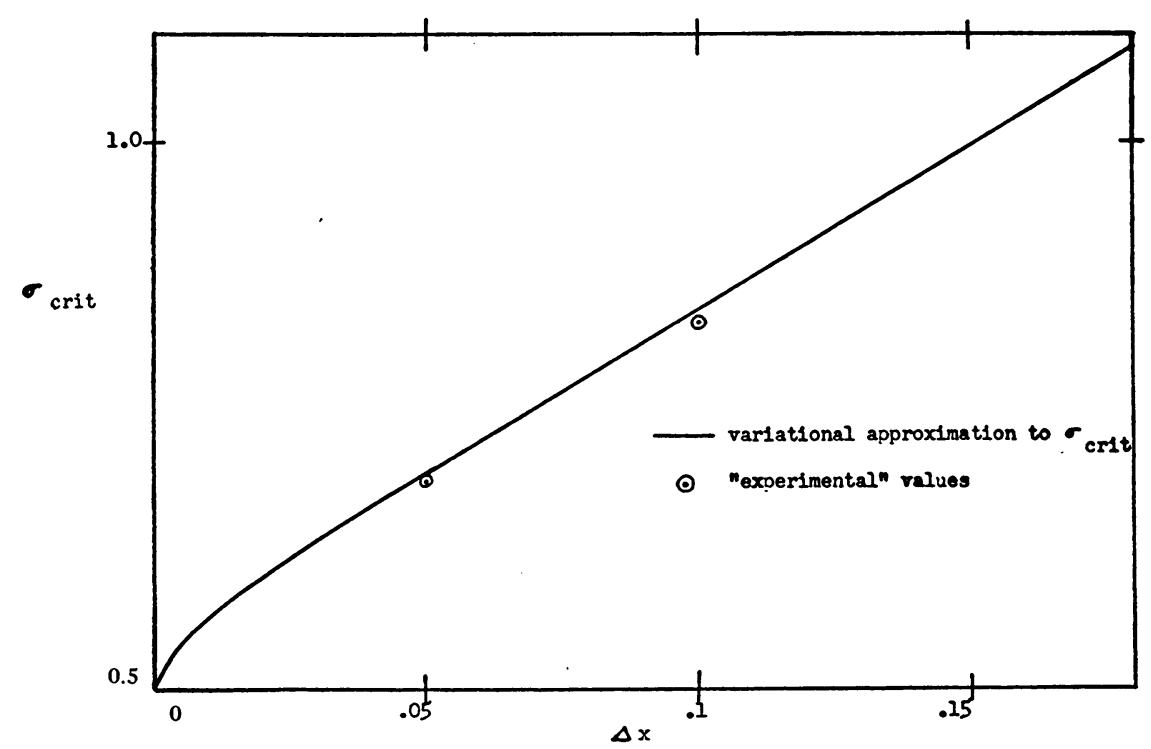

FIG. 1.

To illustrate the uncomfortable limitation imposed by the stability condition we assume (arbitrarily) that the calculation is to be carried to sufficiently great $t$ that $\phi(r, t)$ at $r=100$ is substantially affected by the inner boundary value, $a$. This requires $t_{\max } \sim(100)^{2}$. To represent adequately the structure of $\phi$ near $r=1$ requires, say, $\Delta x=0.1$. Then the stability condition requires

$$
\Delta t \leqslant \sigma_{\text {crit }}(\Delta x)^{2}=0.0085 .
$$

The number of "cycles" ( $j$-values) required for the calculation is then $t_{\max } / \Delta t \sim 10^{6}$. A moderately fast electronic digital computer could complete the calculation in a few hundred hours!

4. A generally stable difference equation. The difference equation (5) determines a set of numbers, $\phi_{n j}$, which approximate the solution of the 
differential equation (4) at the junction points of the rectangular lattice $x=n \Delta x, t=j \Delta t$. The alternative difference equation here considered makes use of a diagonal lattice, obtained by omitting those junction points for which $n+j$ is, say, odd. The term $\phi_{t}$ is now represented by the difference of two $\phi_{n j}$-values of the same $n$ and $j$ 's differing by 2 ; i.e. $\phi_{t}(n \Delta x, j \Delta t) \sim$ $\left(\phi_{n, j+1}-\phi_{n, j-1}\right) / 2 \Delta t ; n+j$ odd. In the representation of $\phi_{x x}$ by a second difference with respect to $n$ the end terms, $\phi_{n-1, j}$ and $\phi_{n+1, j}$, may be used as previously but the term, $\phi_{n, j}$, corresponds to a point omitted from the lattice. It is therefore replaced by the mean of the two terms of neighboring $j$-values.

$$
\phi_{x x}(n \Delta x, j \Delta t) \sim\left(\phi_{n-1, j}-\phi_{n, j-1}-\phi_{n, j+1}+\phi_{n+1, j}\right) /(\Delta x)^{2} ; n+j \text { odd. }
$$

The resulting difference approximation to (4) is

$$
\begin{aligned}
\phi_{n, j+1}-\phi_{n, j-1}=2 p_{n} \sigma\left(\phi_{n-1, j}-\phi_{n, j-1}-\phi_{n, j+1}+\phi_{n+1, j}\right), \\
n+j \text { odd, } \quad p_{n}>0, \quad \sigma=\Delta t / \Delta x^{2}
\end{aligned}
$$

which may be written as

$$
\phi_{n, j+1}=\phi_{n, j-1}+\alpha_{n}\left(\phi_{n-1, j}-2 \varphi_{n, j-1}+\phi_{n+1, j}\right),
$$

where

$$
\alpha_{n}=2 p_{n} \sigma /\left(1+2 p_{n} \sigma\right) ; \quad 0<\alpha_{n}<1 .
$$

If the differential equation has terms in $\phi_{x}$ and $\phi$ these may be represented by $\left(\phi_{n+1, j}-\phi_{n-1, j}\right) / 2 \Delta x$ and $\left(\phi_{n, j+1}+\phi_{n, j-1}\right) / 2$ respectively.

It is to be noted that the initial conditions required to permit calculation with (13) require the specification of $\phi$-values for two initial times ( $j$-values), or initial boundaries, $x(t)$, when only one would be required by the conventional difference equation. This requirement suggests that (13) is of hyperbolic rather than parabolic character (cf. section 5). The second set of initial values may be computed from the first set by use of the conventional equation with sufficiently small $\Delta t$.

In each cycle of the calculation $\phi_{n, j}$ may be evaluated for all $n$-values associated with one $j$-value, even $n$ occurring in one cycle, odd $n$ in the next. Alternatively the $\phi_{n, j}$ may be evaluated in each cycle for all $n$ along a diagonal line $n+j=$ constant (or $n-j=$ constant). These two orderings of the calculation, called the "leap-frog" and "pyramid" methods respecively, are shown in Fig. 2.

In the pyramid method the cycle number may be used to index the $\phi$-values in place of $j$. If the cycle number, $m$, is written as a super-script, the difference equation (13) takes the form

$$
\phi_{n}^{m+1}=\phi_{n}^{m}+\alpha_{n}\left(\phi_{n-1}^{m+1}-2 \phi_{n}^{m}+\phi_{n+1}^{m}\right) .
$$

This form of the difference equation displays clearly the similarity to the "extrapolated Liebmann" form of the relaxation method. ${ }^{2}$

The qualitative behavior of the solution of (13) may be investigated by examining the propagation of a spatially sinusoidal component of $\varphi$, treating $p_{n}$, hence also $\alpha_{n}$, as a constant. If

$$
\lambda_{n, j}=K^{i} e^{i n \gamma}
$$


satisfies (13) for constant $\alpha_{n}$ then

Hence

$$
K=K^{-1}+\gamma\left(e^{-i \gamma}-2 K^{-1}+e^{i \gamma}\right) .
$$

$$
K^{2}-2 K \alpha \cos \alpha+(2 \alpha-1)=0 .
$$

For each $\gamma$, two values of $K$ satisfy (15). Thus a sinusoidal component of $\phi$ has two modes of propagation with increasing $t$. The propagation factors, $K$, are shown in Fig. 3. For $\gamma$ near 0 or near $\pi$ the propagation factors are
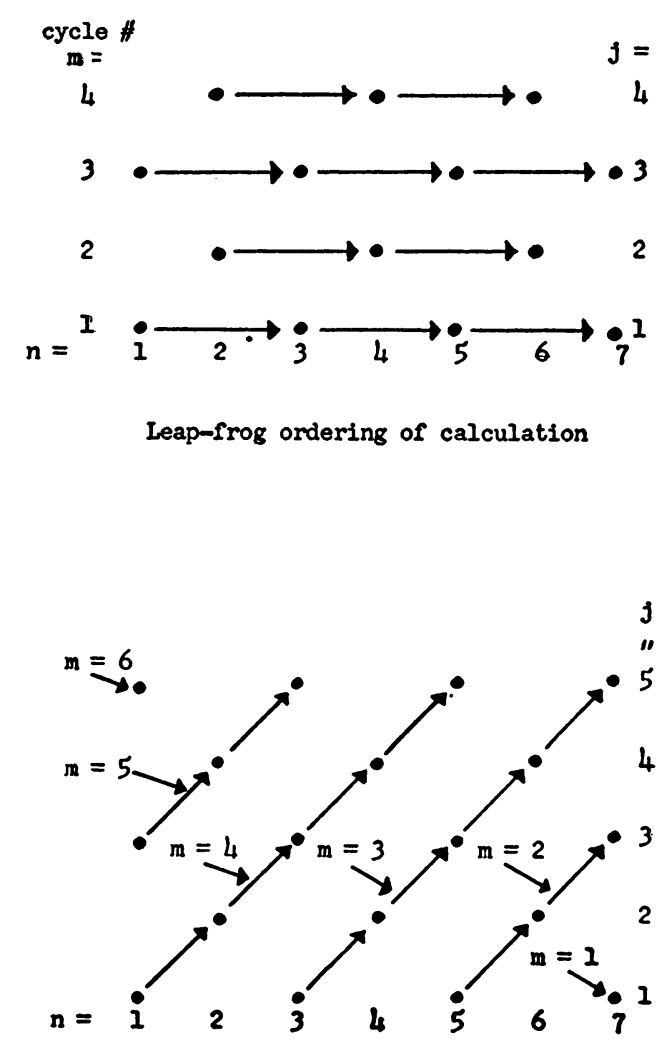

Pyramid ordering of calculation

$(n-j)=$ constant

FIG. 2.

real, for intermediate $\gamma$ they are complex. In no case is the magnitude of $K$ greater than unity. The difference equation (13) is thus seen to be stable for any constant positive value of $p_{n} \sigma$.

To show the stability of (13) for a varying $\alpha_{n}$, we assume the presence of an error-eigenfunction of the form

$$
\phi_{n, j}=K^{\lambda} \lambda_{n} ; \quad \lambda_{0}=\lambda_{N}=0 .
$$


Substitution in (13) yields

$$
\lambda_{n-1}+\lambda_{n}\left(1-K^{2}-2 \alpha_{n}\right) / K \alpha_{n}+\lambda_{n+1}=0
$$

or

$$
\omega^{2}\left(2 \lambda_{n}-\lambda_{n-1}-\lambda_{n+1}\right)-4 \omega\left(\alpha_{n}^{-1}-1\right) \lambda_{n}+\left(2 \lambda_{n}+\lambda_{n-1}+\lambda_{n+1}\right)=0,
$$

where

$$
K=(\omega-1) /(\omega+1) .
$$

Multiplying (16) by $\lambda_{n}{ }^{*}$ and summing over $n=1,2, \cdots, N-1$ produces, after slight rearrangement of terms, the equation

$$
\omega^{2} \sum_{1}^{N}\left|\lambda_{n}-\lambda_{n-1}\right|^{2}-4 \omega \sum_{1}^{N-1}\left(\alpha_{n}^{-1}-1\right)\left|\lambda_{n}\right|^{2}+\sum_{1}^{N}\left|\lambda_{n}+\lambda_{n-1}\right|^{2}=0 .
$$

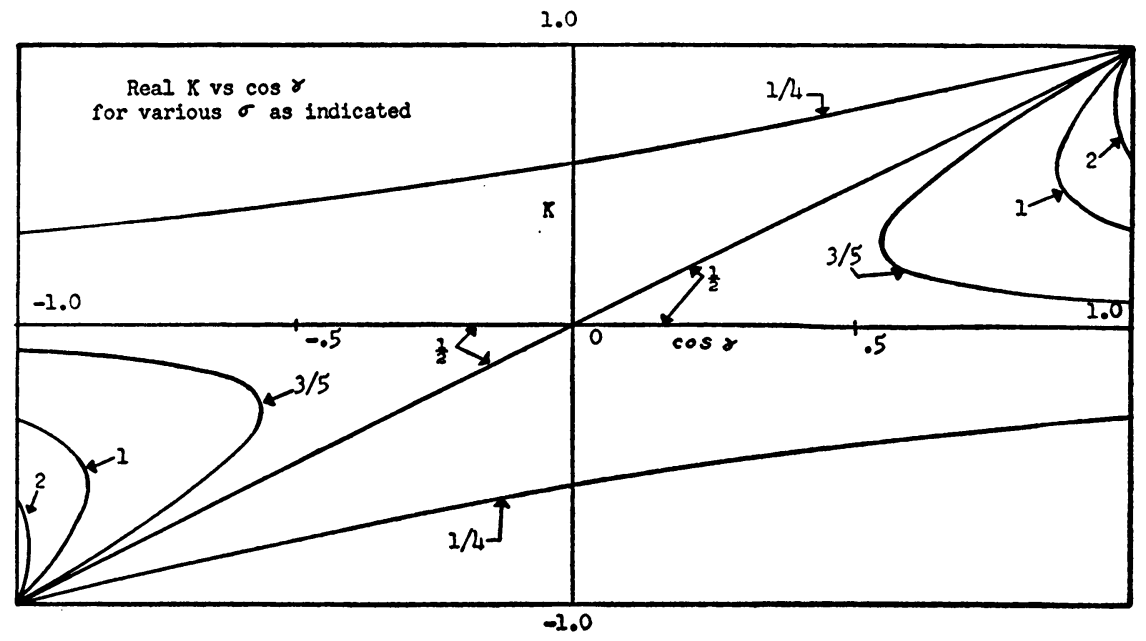

FIG. 3.

The positiveness of the three summations ensures that the real part of $\omega$ is positive, hence by (17)

$$
|K|<1 \text {. }
$$

If the fixed end conditions, $\lambda_{0}=\lambda_{n}=0$, are replaced by the homogeneous boundary conditions,

$$
\begin{aligned}
\lambda_{1}-\lambda_{0} & =A \lambda_{0} \\
\lambda_{N-1}-\lambda_{N} & =B \lambda_{N}
\end{aligned} \quad A \text { and } B \text { real and positive, }
$$

equation (18) is modified by the inclusion of additional positive terms in the first and third summations. Then again (19) follows from the positiveness of $\left(\alpha_{n}^{-1}-1\right)$; hence the difference equation (13) is stable for any positive $\sigma$.

5. Dependence of the solution on the lattice spacings. To illustrate the dependence of the difference equation (13) and its solution on the lattice intervals, $\Delta x$ and $\Delta t$, we examine in detail the special case of constant $p(x)$. 
This constant may be given the value unity without loss of generality. The difference equation is then

$$
\phi_{n, j+1}-\phi_{n, j-1}=2 \sigma\left(\phi_{n-1, j}-\phi_{n, j-1}-\phi_{n, j+1}+\phi_{n+1, j}\right), \sigma=\Delta t / \Delta x^{2},
$$

which approximates the homogeneous, one-dimensional diffusion equation

$$
\phi_{t}=\phi_{x x} .
$$

If the set of four $\phi$-values connected by (20) are assumed to be imbedded in a function $\phi(x, t)$ which permits a power series expansion about the "center point" $(n, j)$, they may be described by the expansions

$$
\begin{aligned}
& \phi_{n \pm 1, j}= \phi \pm \Delta x \phi_{x}+\frac{1}{2}(\Delta x)^{2} \phi_{x x} \pm \frac{1}{6}(\Delta x)^{3} \phi_{x x x}+\frac{1}{24}(\Delta x)^{4} \phi_{x x x x}+\cdots \\
& \phi_{n, j \pm 1}=\phi \pm \Delta t \phi_{t}+\frac{1}{2}(\Delta t)^{2} \phi_{t t} \pm \frac{1}{6}(\Delta t)^{3} \phi_{t t t}+\frac{1}{24}(\Delta t)^{4} \phi_{t t t t}+\cdots
\end{aligned}
$$

Substitution into (20) then yields

$$
\begin{aligned}
\phi_{t}-\phi_{x x}=\frac{1}{12}(\Delta x)^{2} \phi_{x x x x}-(\Delta t)^{2} \phi_{t t} /(\Delta x)^{2} & -\frac{1}{6}(\Delta t)^{2} \phi_{t t t} \\
& -\frac{1}{12}(\Delta t)^{4} \phi_{t t t t} /(\Delta x)^{2}+\cdots
\end{aligned}
$$

The use of (20) as an approximation to the diffusion equation thus consists in the neglect of the right member of (22). The use of small values of $\Delta x$ and $\Delta t$ is not alone sufficient to justify this neglect. To make the second term of the right member of (22) negligible requires also that the ratio, $\Delta t / \Delta x$ be small. If $\Delta x$ and $\Delta t$ approach zero with constant ratio, $c=\Delta x / \Delta t$, the difference equation (20) does not approximate the parabolic equation (21), but rather the hyperbolic equation

$$
\phi_{t t} / c^{2}+\phi_{t}=\phi_{x x}
$$

It may be noted that the mesh ratio, $\Delta t / \Delta x$, is just the maximum permitted by the stability condition for the treatment of this hyperbolic equation by the usual difference method. The stability of the difference equation (20) may be regarded as arising from the introduction of this hyperbolic term in the approximating differential equation.

The above considerations indicate that the requirement for accuracy of treatment of the parabolic equation approximated by (20) is the smallness of both $\Delta x$ and $\Delta t / \Delta x$. The ratio, $\Delta t / \Delta x$ is not, however, required to be small in the order of $\Delta x$, as would be needed for a fixed value of $\sigma$. Since (21) implies

$$
\phi_{t t}=\phi_{x x x x}
$$

the dominant terms in the right member of (22) may be written

$$
\phi_{t}-\phi_{x x}=\frac{1}{12}(\Delta x)^{2} \phi_{x x x x}\left(1-12 \sigma^{2}\right)+\cdots .
$$

Thus for fixed $\Delta x$ the optimum value for $\Delta t$, from considerations of accuracy alone, lies near $12^{-\frac{1}{3}} \Delta x^{2}$. 
The accuracy of the difference equation representation of (21) may be examined by reference to the Fourier expansion used in establishing its stability. A component of $\phi$ having the sinusoidal dependence, $e^{i n \gamma}$, is exponentially attenuated with increasing $j$ by the factor $K$ per cycle. The attenuation factor, $K$, is determined by (15) and is double valued (again suggesting the hyperbolic character of the difference equation). As shown in Fig. $4, K_{+}$, the greater value of $K$, closely approximates the correct attenuation factor, $K_{c}=e^{-\gamma^{2} \sigma}$ (i.e. the factor by which a sinusoidal component $e^{i \gamma x / \Delta x}$ is attenuated in time $\Delta t$ by the differential equation (21)) for small $\gamma$. With increasing $\gamma$ this greater $K$ becomes progressively more unrealistic,

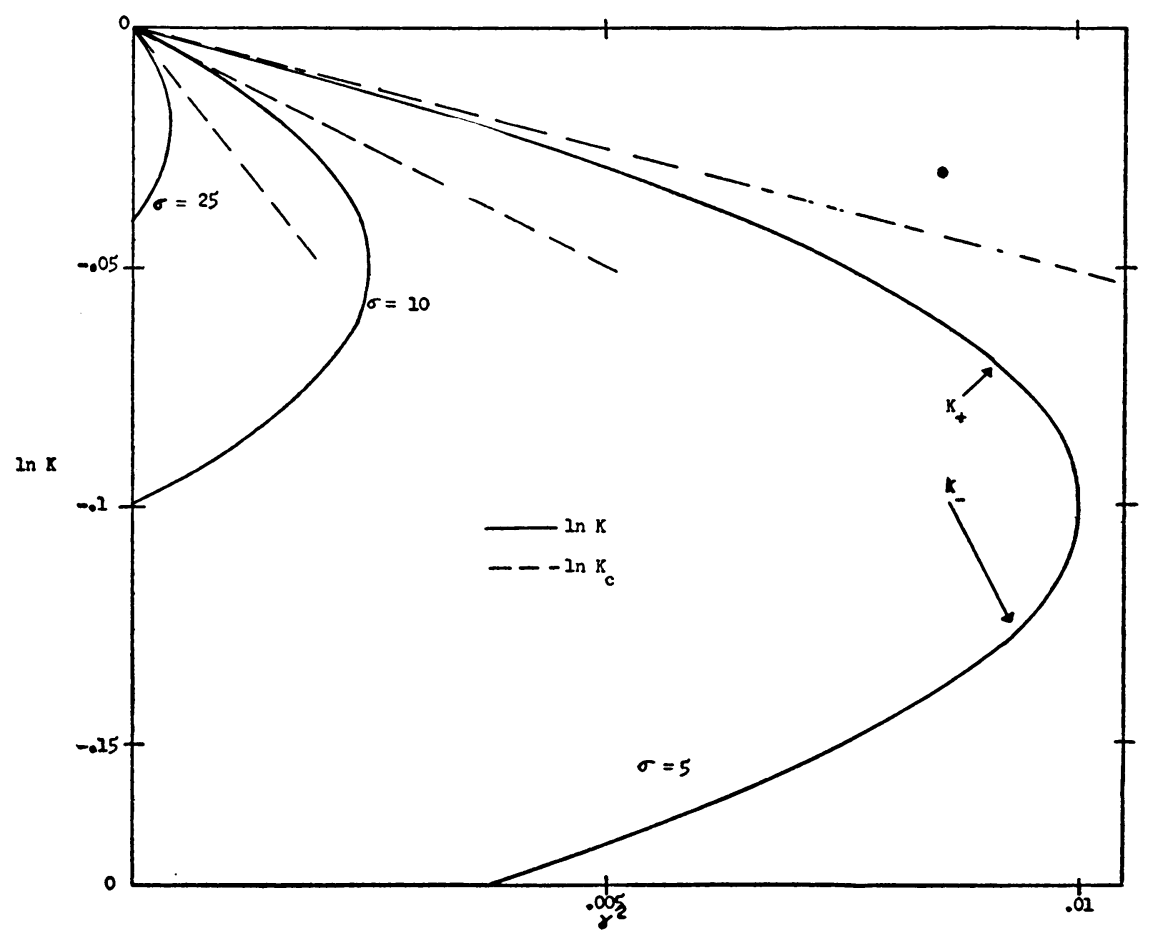

FIG. 4.

the more rapidly the greater is $\sigma$. For

$$
0 \leqslant \cos \gamma<\left(1-1 / 4 \sigma^{2}\right)^{\frac{1}{2}}
$$

the two $K$ 's become complex, of common magnitude $[(2 \sigma-1) /(2 \sigma+1)]$. For negative values of $\cos \gamma$ the attenuation factors are the negatives of those for the corresponding positive $\cos \gamma$. Since only even values of $n+j$ are considered these negative values of $\cos \gamma$ are superrogatory.

The rapid increase of the error in $K_{+}$with increasing $\gamma$ would seem to indicate that the difference equation (20) is of unacceptable accuracy except for small $\sigma$. After a large number of cycles of calculation (large $j$ ), however, the two values, $K_{+}$and $K_{c}$, appear in the solutions of (20) and (21) raised to the power $j$. For sufficiently large $j$, both $K_{+}{ }^{j}$ and $K_{c}{ }^{i}$ are essentially zero 
except for very small $\gamma$. An approximate measure of error is thus the greatest value of the discrepancy,

$$
D_{j}=K_{c}{ }^{j}-K_{+}{ }^{i}=e^{-j \sigma \gamma^{2}}-K_{+}{ }^{j} .
$$

The solution of (15) in power series in $\gamma^{2}$ yields

$$
K_{+}=K_{c} \exp \left[-\gamma^{4}\left(\sigma^{3}-\sigma / 12\right)+\cdots\right] .
$$

For $j \gg 4(\sigma-1 / 12 \sigma)$ the greatest discrepancy occurs at $\gamma^{2} \cong 2 / j \sigma$ and has the value

$$
D_{j} \cong 4 e^{-2}(\sigma-1 / 12 \sigma) / j .
$$

The difference equation (20) thus permits increasingly accurate representation of the solution of the differential equation (21) as $j$ becomes large in comparison with $4 \sigma$.

The above estimate of accuracy takes into account only the errors in the greater attenuation factor $K_{+}$. An additional error may arise from the failure of the initial specification of $\phi_{n,}$, -values to associate the correct amplitude with the components which decay with attenuation factors, $K_{+}$. Since initial $\phi$-values must be specified for two successive $j$-lines it is not sufficient that the $\phi$ 's closely represent the initial values of the corresponding parabolic differential equation. It is also necessary that the initial time derivative be well represented. The latter requirement is the more stringent the larger is $\sigma$, since $K_{+}$and $K_{-}$are then closer together, hence more difficult to discriminate in the initial-value specification. The value $\sigma=\frac{1}{2}$ (hence $\alpha=\frac{1}{2}$ ) is especially favorable in this regard, since for it $K_{-}$is identically zero (cf. eq. (15)). The special property of $\alpha=\frac{1}{2}$ is also to be seen in (13). For $\alpha=\frac{1}{2}$ the terms in $\phi_{n, j-1}$ drop out, hence specification of initial $\phi$-values for one $j$-line suffices.

Apart from the special case of constant $p_{n}$, no choice of $\sigma$ makes $\alpha_{n}$ uniformly equal to $\frac{1}{2}$. It nevertheless seems expedient to begin the calculation with a $\sigma$ which makes $\frac{1}{2}$ a typical value for $\alpha_{n}$. After a sufficient number of cycles $\sigma$ can conveniently (i.e. without requiring interpolation) be increased by an odd integral factor.

6. Examples of the use of the generally stable difference equation. The first example chosen to illustrate the use of the difference equation (13) is the equation of diffusion to the boundary of a one-dimensional semi-infinite medium. Its differential equation and boundary conditions are

$$
\begin{gathered}
\phi_{t}=\phi_{x x} \text { for } t>0, \quad 0<x<\infty, \\
\phi(x, 0)=1, \quad \phi(0, t)=0 .
\end{gathered}
$$

It has the simple solution

$$
\phi(x, t)=\operatorname{erf}\left(x / 2 t^{\frac{1}{3}}\right) .
$$

Numerical solutions were obtained by the pyramid method for two values of $\sigma, \frac{1}{2}$ and 5 . The boundary values are

$$
\phi_{0}{ }^{m}=0, \quad \phi_{n}{ }^{0}=1 \text { for } n>0 .
$$

Subsequent $\phi$-values are determined by the relation

$$
\begin{aligned}
\phi_{n}^{m+1} & =\phi_{n}^{m}+\alpha_{n}\left(\phi_{n+1}^{m+1}-2 \phi_{n}^{m}+\phi_{n-1}^{m}\right), \\
\alpha_{n} & =\frac{1}{2} \text { or } 10 / 11 \text { as } \sigma=\frac{1}{2} \text { or } 5 .
\end{aligned}
$$


For each cycle ( $m$-value) the $\phi_{n}^{m+1}$ are computed for successively smaller $n$, beginning with an $n$ sufficiently large that $\phi_{n}^{m+1}$ does not differ appreciable from unity. (Here $2 m=n+j$.)

The resulting $\phi$-values are displayed in Fig. 5 and 6, plotted for several values of $t=\Delta t(2 m-n)$ vs. the similarity variable,

$$
\mu=x / 2 t^{\frac{3}{2}}=n / 2[\sigma(2 m-n)]^{\frac{1}{2}} \text {. }
$$

The approach of $\phi_{n}{ }^{m}$ to $\phi(\mu)$ with increasing $j=2 m-n$, hence $a$ fortiori with increasing $t=\sigma j \Delta x^{2}$, is noticeably more rapid for the smaller $\sigma$.

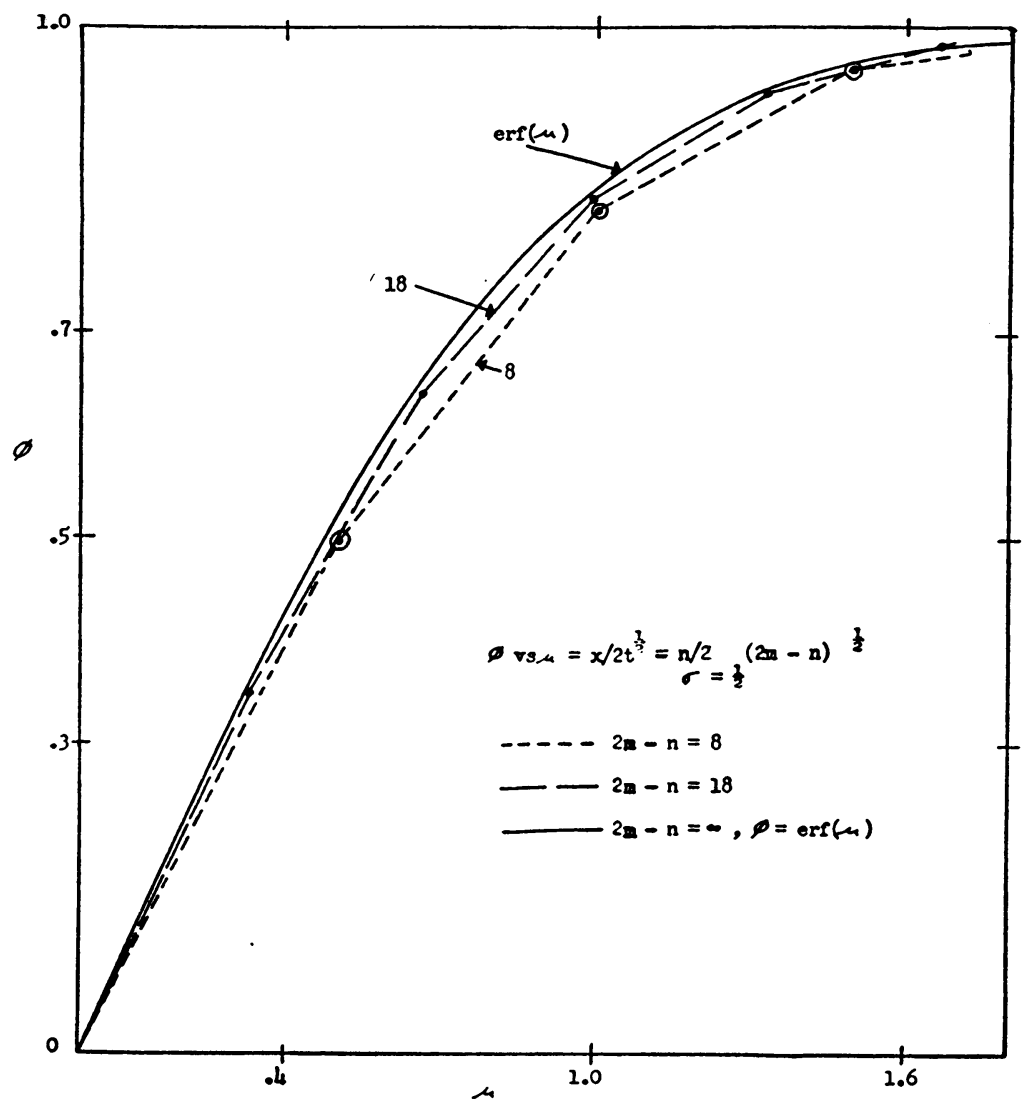

Fig. 5. Results of One Dimensional Diffusion Problem

A second example used is the radial diffusion equation,

$$
\phi_{t}=\phi_{r r}+\phi_{r} / r ; \quad r \geq 1 \text {. }
$$

Setting $r=e^{x}$ permits writing (26) in the form

$$
\phi_{t}=e^{-2 x} \phi_{x x} ; x \geq 0 \text {. }
$$

The boundary values

were chosen.

$$
\phi(0, t)=0, \quad \phi(x, 0)=1
$$


Again the pyramid ordering was used; hence the difference equation is (25) with

$$
\alpha_{n}=2 \sigma e^{-2 n \Delta x} /\left(1+2 \sigma e^{-2 n \Delta x}\right) .
$$

The boundary conditions of the difference equation are given again by (24). Three solutions were obtained, with intervals as follows:

\begin{tabular}{lllc} 
Case & $\Delta x$ & \multicolumn{1}{c}{$\Delta t$} & $\sigma=\Delta t / \Delta x^{2}$ \\
I & .05 & .00125 & $\frac{1}{2}$ \\
II & .05 & .0125 & 5 \\
III & .10 & .05 & 5
\end{tabular}

The solution in Case I shows good accuracy even in the first few cycles. A comparison with an analytically derived solution for $t=1 / 100=8 \Delta t$ is shown in Fig. 7. The four points plotted are obtained in the fifth, sixth, seventh, and eighth cycles respectively.

A somewhat greater number of cycles is required for comparable accuracy in Case II.

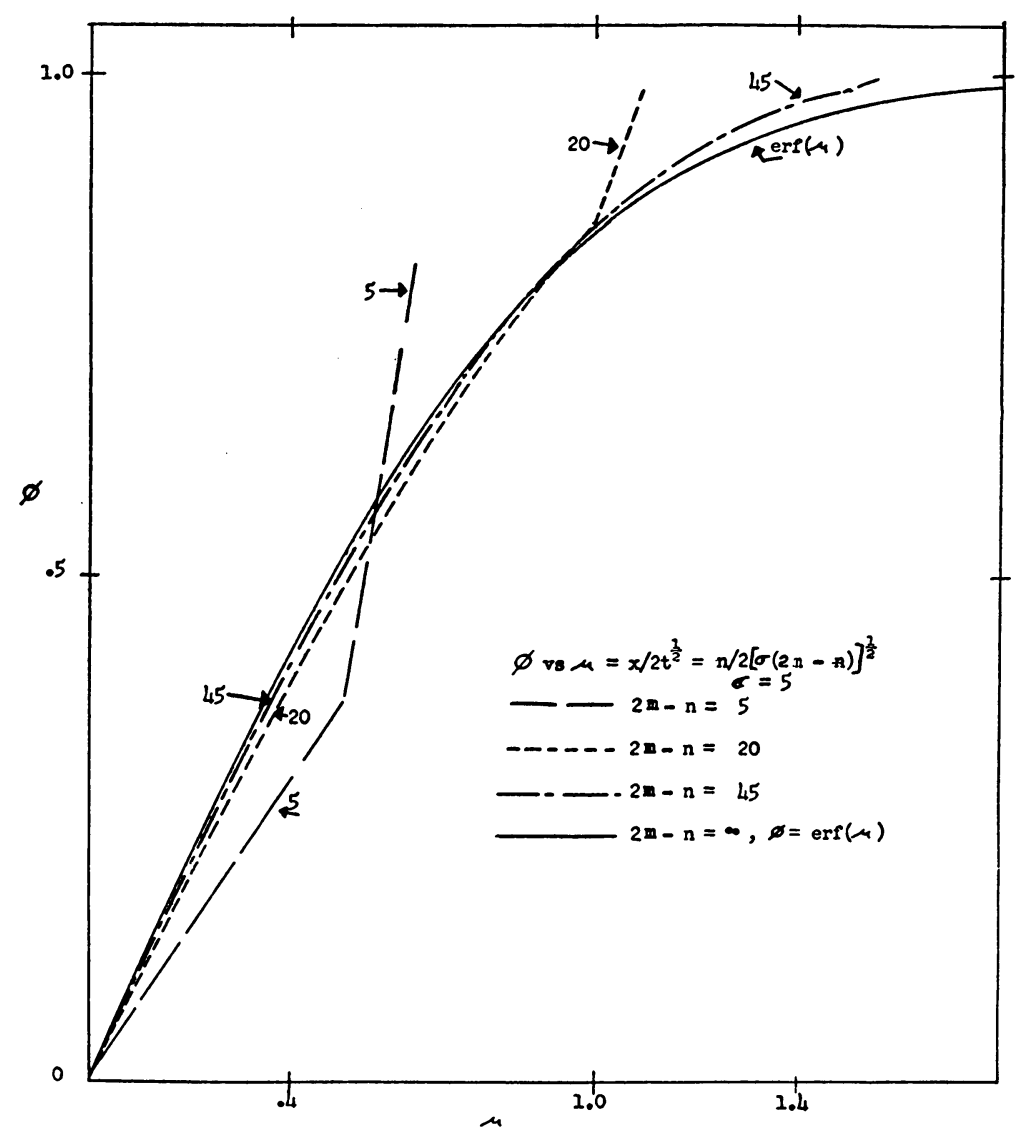

Fig. 6. Results of One Dimensional Diffusion Problem 


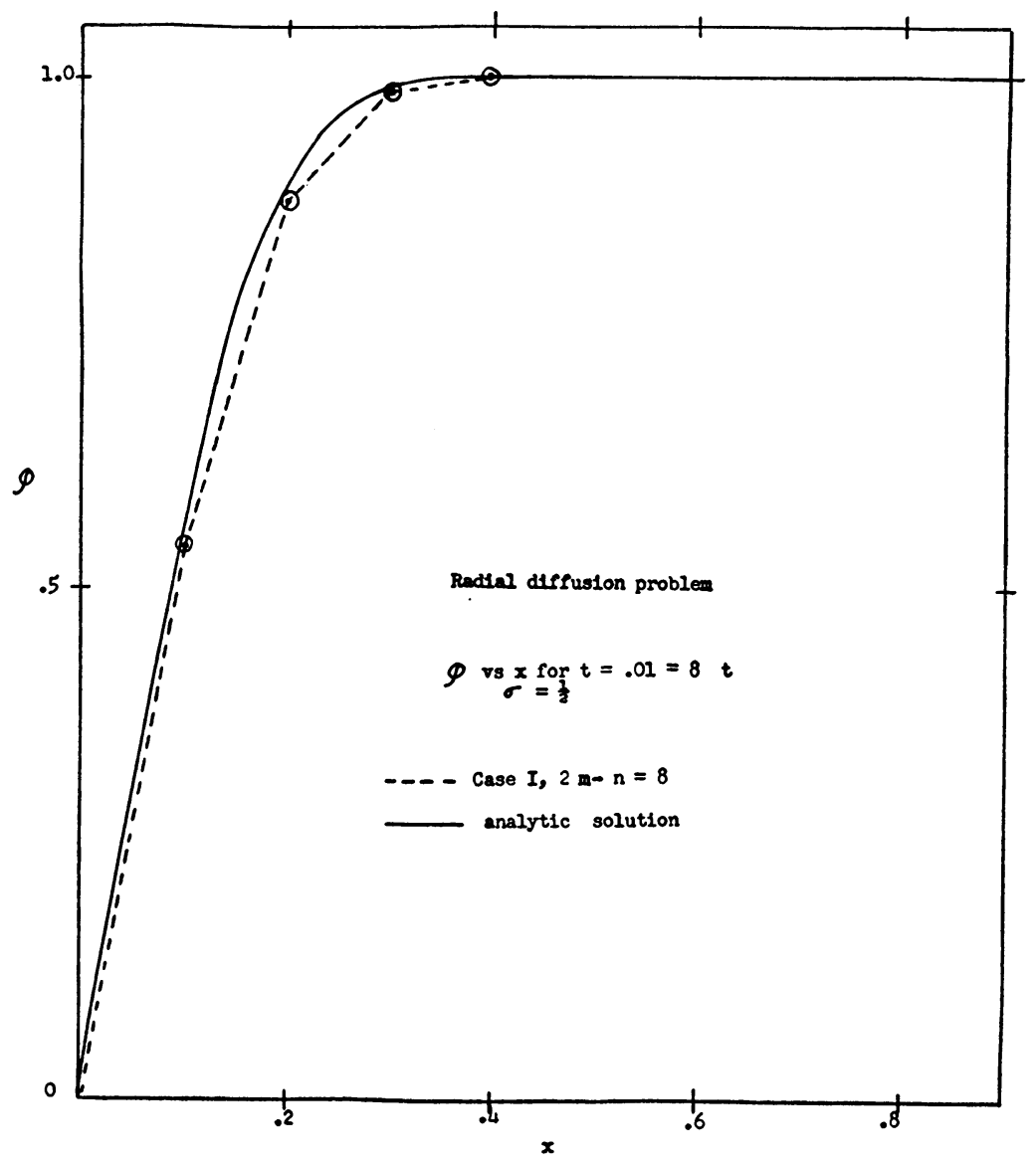

FIG. 7.

Its solution is shown for $t=5 \Delta t$ and $t=17 \Delta t$ in Fig. 8. For the latter time the plotted points are obtained in the ninth and subsequent cycles, and are of about the same accuracy as the points in Fig. 7.

The solution in Case III is shown in Fig. 9 for $t=5 \Delta t, 15 \Delta t$, and $40 \Delta t$. The values for $40 \Delta t=2.0$ (derived from the twenty-first and subsequent cycles) are not distinguishable in this graph from the previous cases nor from the analytic solution.

7. Extension to other parabolic equations. The demonstration given above of the stability of the leap-frog (or pyramid) method depends upon an eigenfunction expansion of the errors in the dependent variable. (So, in fact, does the definition of stability there used.) To justify the use of these methods for broader classes of parabolic equations other indications of stability must be sought. In section 8 several types of linear parabolic difference equations are shown to display properties indicative of stability. No uniform definition and proof of stability has been produced, however. 


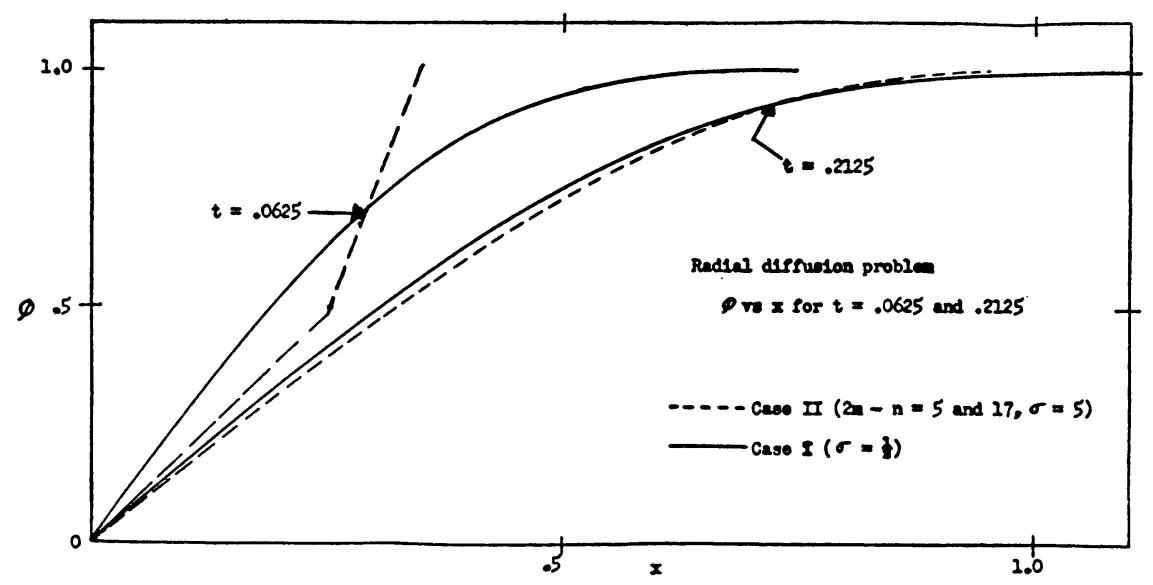

FIG. 8. tion,

A similar computational technique can be applied to a nonlinear equa-

$$
\phi_{t}=f\left(\phi, \phi_{x}, \phi_{x x}, x, t\right) .
$$

In suitable cases $\phi(x, t)$ may be sufficiently well represented by a set of numbers, $\phi_{n, j}$, satisfying the corresponding difference equation,

$$
\begin{array}{r}
\left(\phi_{n, j+1}-\phi_{n, j-1}\right) / 2 \Delta t=f\left[\frac{1}{2}\left(\phi_{n, j+1}+\phi_{n, j-1}\right), \quad \frac{1}{2}\left(\phi_{n+1, j}-\phi_{n-1, j}\right) / \Delta x,\right. \\
\left.\left(\phi_{n+1, j}-\phi_{n, j-1}-\phi_{n, j+1}+\phi_{n-1, j}\right) /(\Delta x)^{2}, \quad n \Delta x, j \Delta t\right] .
\end{array}
$$

The stability of this computational procedure depends upon the rates of growth of small deviations from this "correct" solution. If equation (29) permits a power series development in these deviations then they will, when sufficiently small, be governed by a linear parabolic difference equation of

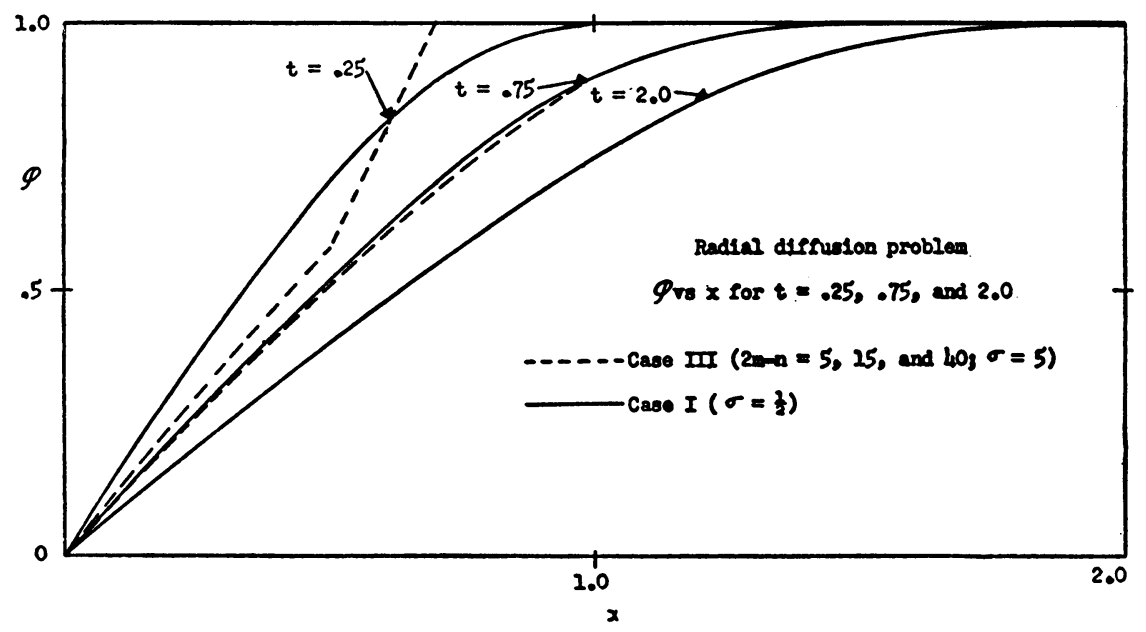

FIG. 9. 
the same form. The above stability arguments for linear equations thus indicate a wide range of usefulness for this procedure as applied to nonlinear equations.

To illustrate this procedure we consider the equation describing the onedimensional isothermal flow of a perfect gas in a porous medium.

$$
\begin{gathered}
\phi_{t}=\left(\phi^{2}\right)_{x x} \text { for } t>0, \quad x>0 \\
\phi(x, 0)=1 ; \quad \phi(0, t)=0 .
\end{gathered}
$$

A convenient corresponding difference equation is

$$
\Delta \phi \equiv \phi_{n, j+1}-\phi_{n, j-1}=2 \sigma\left[\phi_{n-1, j}^{2}-2 \phi_{n, j-1}^{2}+\phi_{n+1, j}^{2}-\Delta \phi\left(\phi_{n-1, j}{ }^{+} \phi_{n+1, j}\right)\right] .
$$

The use of $\phi_{n-1, j}+\phi_{n+1, j}$ rather than $\phi_{n, j-1}+\phi_{n, j+1}$ as the coefficient of $\Delta \phi$ in the right member is consistent with the order of approximation to (30)

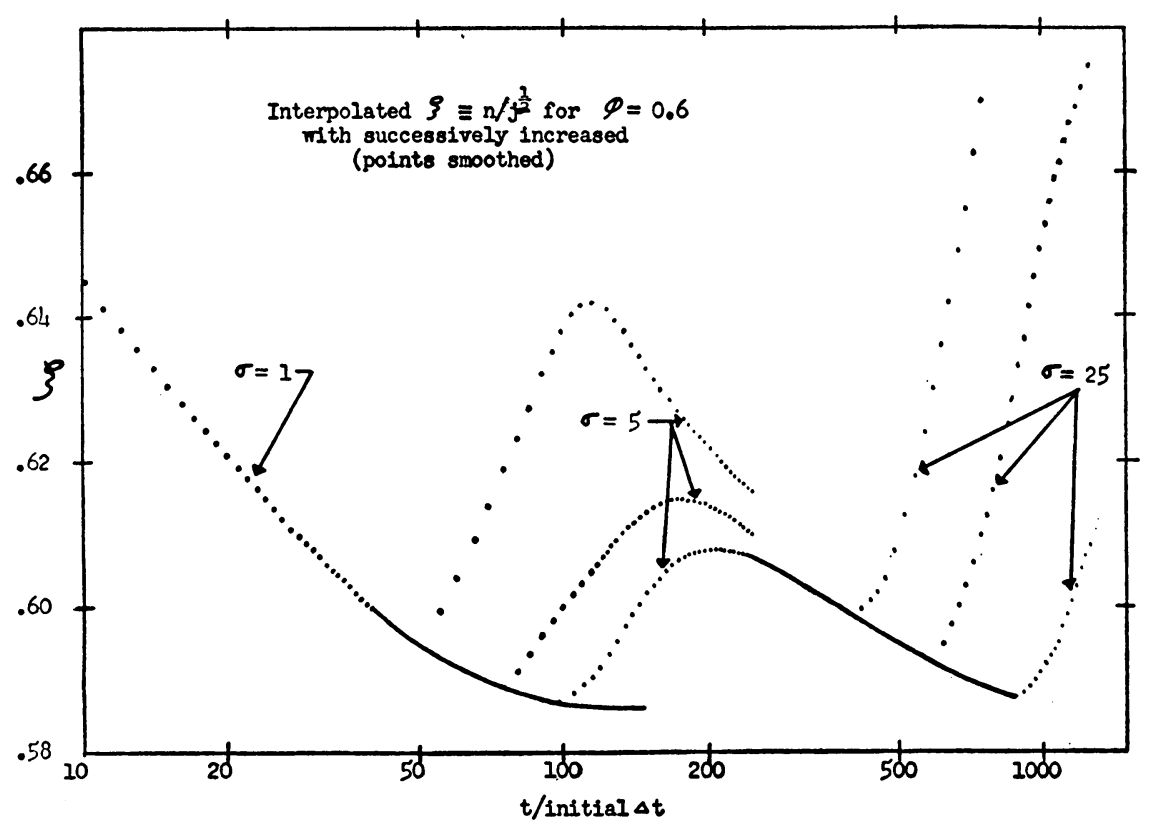

FIG. 10.

used and simplifies the explicit expression for $\phi_{n, j+1}$. This is

$$
\begin{array}{r}
\phi_{n, j+1}=\phi_{n, j-1}+2 \sigma\left[\phi^{2}{ }_{n-1, j}-2 \phi^{2}{ }_{n, j-1}+\phi_{n+1, j}\right] \\
/\left[1+\sigma\left(\phi_{n-1, j}+\phi_{n+1, j}\right)\right] .
\end{array}
$$

Solutions to (31) were obtained with $\sigma$ initially given the value 1 , later increased by successive factors of five. (An attempt to start a solution with $\sigma=5$ led to uncontrolled oscillation.) When $\sigma$ was not increased too soon, qualitatively correct solutions were obtained. The solution of (30) is of the form $\varphi\left(x / t^{t}\right)$. Accordingly, to provide a measure of accuracy of the approximation of $\phi_{n, j}$ to the solution of (30), the $\phi_{n, 5}$ values for each cycle were used to determine a graphically interpolated value of $\xi=n / j^{\sharp}$ corresponding 
to the arbitrarily selected value, $\phi_{n, j}=0.6$. The discrepancy between $\xi$ and its asymptote, approximately 0.586 , indicates the order of inaccuracy of the solution. Fig. 10 shows values of $\xi$ for a family of solutions of (31) differing in the number of cycles carried out at each value of $\sigma$. The abscissa, displayed logarithmically, shows $t$ in units of the initial $\Delta t$ rather than the number of cycles of calculation performed. The error (of approximation to the solution of (30)) is seen to increase and later subside slowly after each increase in $\sigma$. The peak error decreases rapidly with deferral of the increase in $\sigma$. To keep the error of the order of one percent requires a few hundred cycles at each $\sigma$-value, somewhat more in the later stages.

8. Stability for the general parabolic linear equation. The differential equation,

$$
\phi_{t}=p(x, t) \phi_{x x}+q(x, t) \phi_{x}+r(x, t) \phi
$$

may be approximated by the difference equation

$$
\begin{aligned}
& \left(\phi_{n, j+1}-\phi_{n, j-1}\right) / 2 \Delta t=p_{n j}\left(\phi_{n+1, j}-\phi_{n, j+1}-\phi_{n, j-1}+\phi_{n-1, j}\right) / \Delta x^{2} \\
& \quad+q_{n j}\left(\phi_{n+1, j}-\phi_{n-1, j}\right) / 2 \Delta x+r_{n j}\left(\phi_{n, j+1}+\phi_{n, j-1}\right) / 2 ; n+j \text { odd }
\end{aligned}
$$

where the notation and lattice structure is as described above. The solution of (33) may be expected to provide a reasonable approximation to that of (32) only if throughout the region considered

$$
\begin{aligned}
p & \geqslant 0 \\
|q \Delta t / \Delta x| & \leqslant 1, \quad \text { and } \\
|r \Delta t| & \ll 1 .
\end{aligned}
$$

It is assumed here that initial rather than final values of $\phi$ are specified; hence (34) is necessary for the uniqueness of the solution. Condition (35) is clearly required by considerations of causality similar to those described above. (i.e., the lattice slope, $|\Delta x / \Delta t|$, must clearly be at least as great as the "stream velocity," $|q|$.) It ensures that the cone of determination of each $\phi_{n j}$ includes the characteristic curve of (32), satisfying

$$
d x / d t=-q(x, t)
$$

and passing through $(n \Delta x, j \Delta t)$. In the event $p=q=0$, (32) becomes an ordinary differential equation (involving $x$ as a parameter) which is reasonably approximated by (33) only if (36) is satisfied.

We first consider the case $q \equiv r \equiv 0$. Equation (33) then reduces to

$$
\left(\phi_{n, j+1}-\bar{\phi}_{n j}\right)=\left(\phi_{n, j-1}-\bar{\phi}_{n j}\right)\left(1-2 p_{n j} \sigma\right) /\left(1+2 p_{n j} \sigma\right)
$$

where

$$
\bar{\phi}_{n j}=\frac{1}{2}\left(\phi_{n-1, j}+\phi_{n+1, j}\right) \text { and } \sigma=\Delta t / \Delta x^{2}
$$

hence

$$
\left|\phi_{n, j+1}-\bar{\phi}_{n j}\right| \leqslant\left|\phi_{n, j-1}-\bar{\phi}_{n j}\right| .
$$

Each stage of the computation is characterized by a "front" in the $n, j$ lattice bounding the region for which $\phi$-values have been computed. The front is specified by a single-valued function, $j(n),(j+n$ even $)$, satisfying 
$j(n+1)=j(n) \pm 1$. For $j \leqslant j(n), \phi_{n j}$ has been computed, otherwise not. In each step of the calculation a "low" point of the front, $j(n)=j(n \pm 1)-1$, is replaced by $j(n)+2$. Thus a "valley" in the front is replaced by a "hill." We define a function of the front consisting of the sum of squares of the first difference of $\phi$, taken along the front:

$$
F=\sum_{n}\left(\phi_{n, j(n)}-\phi_{n-1, j(n-1)}\right)^{2} .
$$

In a step of the calculation two terms of $F$ are altered as a result of the replacement of $\phi_{n, j(n)}$ by $\phi_{n, j(n)+2}$. With the use of (39) it can be shown that this replacement diminishes $F$ unless $p_{n j}=0$ or $\phi_{n, j+1}=\bar{\phi}_{n, j}$, in which event $F$ is unchanged. Thus as the calculation proceeds $F$ decreases monotonically except by reason of influences introduced at the boundaries. This non-increasing character of the (positive) function, $F$, may be regarded as indicating the stability of (33). (Here, and below, the term "stability" is used loosely to indicate the absence of any tendency toward disastrous increase of irregularities in $\phi_{n, j}$ initiated by rounding errors and the like. It is hoped that, pending development of rigorous criteria, these imprecise indications of "stability in the mean" may provide useful hints to the working computer.)

A similar indication of stability can be displayed for the case,

$$
|q \Delta t / \Delta x| \leqslant 1 ;|q \Delta t / \Delta x| \leqslant 2 p \sigma ; \quad r \equiv 0 .
$$

This permits writing (33) in the form:

$$
(1+2 p \sigma) \psi_{+}=(1-2 p \sigma) \psi_{-}+q \Delta t / \Delta x
$$

where

$$
\psi_{+}=\left(\phi_{n, j+1}-\bar{\phi}_{n, j}\right) /\left(\phi_{n+1, j}-\phi_{n-1, j}\right) ; \quad \psi_{-}=\left(\begin{array}{c}
\left.\phi_{n, j-1}-\bar{\phi}_{n, j}\right) / \\
\left(\phi_{n+1, j}-\phi_{n-1, j}\right) .
\end{array}\right.
$$

Thus either

$$
\left|\psi_{+}\right| \leqslant\left|\psi_{-}\right| \text {or }\left|\psi_{+}\right| \leqslant \frac{1}{2} .
$$

This permits the inference that $\left|\phi_{n, j-1}-\phi_{n-1, j}\right|+\left|\phi_{n+1, j}-\phi_{n, j-1}\right|$ is (in the first event) decreased or (in the second event) at least not increased as a result of the replacement of $\phi_{n, j-1}$ by $\phi_{n, j+1}$. We can thus define a positive front-function

$$
G=\sum_{n}\left|\phi_{n, j(n)}-\phi_{n-1, j(n-1)}\right|
$$

which is non-increasing (except by reason of disturbance at the boundaries). The operation of the difference equation thus tends to bring the $\phi$-distribution on the front to minimum total variation. This property gives a weaker indication of stability than that described above since $G$, unlike $F$, is not typically minimized by a unique $\phi$-value. It nevertheless seems to justify describing the difference equation as stable.

The second restriction of (41) limits the usefulness of this argument if $2 p \sigma$ becomes small. An argument which is then applicable may be made as follows: Define

$$
\begin{array}{cc}
M_{L}=\phi_{n, j-1}-\phi_{n-1, j} ; \quad M_{R}=\phi_{n+1, j}-\phi_{n, j-1} \\
P_{L}=\phi_{n, j+1}-\phi_{n-1, j} ; \quad P_{R}=\phi_{n+1, j}-\phi_{n, j+1} .
\end{array}
$$


Then equation (33), with $r \equiv 0$, may be shown to imply

$$
\begin{aligned}
(1+q \Delta t / \Delta x) P_{R}{ }^{2}+(1-q \Delta t / \Delta x) P_{L}{ }^{2}=(1+q \Delta t / \Delta x) M_{L}{ }^{2} \\
\quad+(1-q \Delta t / \Delta x) M_{R}^{2}-4 p \sigma(1+2 p \sigma)^{-2}\left\{M_{L}+M_{R}\right. \\
\left.+\left(M_{L}-M_{R}\right) q \Delta t / \Delta x\right\}^{2}
\end{aligned}
$$

A front-function which displays a decreasing tendency may thus be written as

$$
H=\sum_{n}(1 \pm q \Delta t / \Delta x)\left(\phi_{n, j(n)}-\phi_{n-1, j(n-1)}\right)^{2}
$$

$$
\text { the } \pm \text { as } j(n-1)=j(n) \pm 1 \text {. }
$$

In (47) $q$ is properly written $q_{n j}$. In (48) it becomes ambiguous, being either $q_{n-1, j(n)}$ or $q_{n, j(n) \pm 1}$. Thus we can only assert that $H$ is non-decreasing except by reason of variations in $q$ with the advance of the front or disturbance introduced at the boundaries.

In equation (33) with nonvanishing $r$, the precise meaning of "stability" is not evident and no demonstration of properties approximating this concept are known to us. A preliminary qualitative examination failed to disclose any indication that variations in $\phi$ can grow to an alarming extent.

California Institute of Technology and Continental Oil Company

California Institute of Technology
E. C. Du Fort

\section{S. P: FRANKEL}

We are grateful for the support and encouragement given this investigation by the Continental Oil Company. We have been aided by numerous discussions with L. Breiman.

${ }^{1} \mathrm{H}$. LEwy, "On the convergence of solutions of difference equations," Studies and Essays Presented to R. Courant on his 60th Birthday. New York, 1948, p. 211-214.

G. G. O'Brien, M. A. Hyman, \& S. A. KaPlan, "A study of the numerical solution of partial differential equations," Jn. Math. Phys., v. 29, 1951, p. 223-251.

F. JoHn, "On Integration of Parabolic Equations by Difference Methods," Comm. Pure Appl. Math., v. 5, 1952, p. 155-211.

R. P. EDDy, Stability in the Numerical Solution of Initial Value Problems in Partial differential Equations. Naval Ordnance Lab., Memorandum 10232, 1949.

${ }^{2} \mathrm{~S}$. P. FRANKEL, "Convergence rates of iterative treatments of partial differential equations," $M T A C$, v. 4,1950 , p. 65-75.

${ }^{3}$ H. S. Carslaw \& J. C. Jaeger, Conduction of Heat in Solids. Oxford, 1947, p. 280-281.

\section{On Over and Under Relaxation in the Theory of the Cyclic Single Step Iteration}

In order to speed up the sometimes very tedious computations in solving equations by the single step method, the device of the so-called "incomplete relaxation" (under or over relaxation) has been often used, although apparently no systematic discussion of this device has been as yet tried. ${ }^{1}$

It may seem, however, that in the case of the "relaxation procedure" the speeding up can be achieved in this way only in special cases, at least in the case of a symmetric positive definite matrix. Indeed, if the progress of the computation is measured by the decrease of a corresponding quadratic form $A\left(\zeta_{k}\right)$ depending on the $k$-th approximating vector $\zeta_{k}$, we have the formula

$$
A\left(\zeta_{k}\right)-A\left(\zeta_{k+1}\right)=\dot{q_{k}}\left(2-q_{k}\right)\left|r_{N_{k}}^{(k)}\right|^{2} / a_{N_{k} N_{k}}
$$

